\title{
ПРОЕКТИРОВАНИЕ СИСТЕМЫ БИЗНЕС-ПРОЦЕССОВ ДЛЯ РАЗРАБОТКИ ФУНКЦИОНАЛЬНОГО ПРЕДСТАВЛЕНИЯ АРХИТЕКТУРЫ КРУПНОГО ПРОМЫШЛЕННОГО ПРЕДПРИЯТИЯ
}

\author{
(c) 2018 Штейнгарт Евгений Александрович \\ Высшая школа управления и бизнеса \\ Институт промышленного менеджмента, экономики и торговли \\ Санкт-Петербургский политехнический университет Петра Великого \\ 195251, г. Санкт-Петербург, ул. Политехническая, д. 29 \\ E-mail: genja.89@mail.ru
(c) 2018 Бурмистров Андрей Николаевич
кандидат экономических наук, доцент \\ Высшая школа управления и бизнеса \\ Институт промышленного менеджмента, экономики и торговли \\ Санкт-Петербургский политехнический университет Петра Великого \\ 195251, г. Санкт-Петербург, ул. Политехническая, д. 29 \\ E-mail: genja.89@mail.ru
}

Проектирование системы бизнес-процессов - один из ключевых этапов создания архитектуры предприятия, позволяющий идентифицировать все остальные сущности современных хозяйствующих субъектов и упорядочить их деятельность для последующей автоматизации и повышения эффективности. При этом создание такой системы бизнес-процессов с высокой степенью объективности для конкретного предприятия, особенно крупного промышленного, является сложной задачей, в том числе из-за отсутствия в научно-методической литературе методик, позволяющих решать подобные задачи.

Ключевые слова: система бизнес-процессов, функциональная область, архитектура предприятия, референтная функциональная модель, иерархическая декомпозиция, классификация.

Актуальность. В современном мире, в эпоху ускорения развития человечества и постоянных вариаций внешней среды, промышленные предприятия для создания и поддержания конкурентных преимуществ должны автоматизировать свою деятельность. Это необходимо и для выстраивания стабильных и взаимовыгодных кооперационных связей с внешними контрагентами.

Для того чтобы эффективно осуществлять процессы автоматизации и последующего взаимодействия современных промышленных предприятий, их деятельность должна быть хорошо упорядочена и представлена в виде максимально стандартных, понятных, полных и прозрачных моделей работы (действий, функций). Для осуществления упорядочения деятельности предприятий в современной “цифровой экономике” используется концепция архитектуры предприятия (модель основного устройства и связей частей системы [1]), первоочередным представлением которой является функциональное - модели различных функциональных областей и бизнес-процессов предприятия. Это обусловлено тем, что получение конкретных результатов (например, продукции с заданными параметрами), которые необходимы для достижения целей промышленного предприятия возможно только за счёт использования процессного подхода. Таким образом, проектирование системы бизнес-процессов (модели действий, работ) в рамках архитектуры конкретного промышленного предприятия, а также их последующее моделирование и регламентация - актуальная общая научная задача, которая должна решаться с использованием обоснованных максимально стандартизированных методов и инструментов (понятных различным заинтересованным сторонам - от рядовых сотрудников до топ-менеджеров и владельцев бизнеса).

Проблема. Наибольшую сложность представляет разработка системы бизнес-процессов для крупных промышленных предприятий, отличающихся большим количеством работающих 
на них сотрудников (со среднесписочной численностью более 250 человек [2]) и существенными объёмами выпуска продукции (выручка в год больше 2 млрд. рублей [3]). Это ведёт к большому количеству осуществляемых функций [4], сложности их упорядочения (структурирования) и максимально высокой степени влияния субъективных качеств разработчика на создаваемую модель. Кроме этого, для отечественных предприятий дополнительные сложности накладывают международные санкции, которые повышают риски использования зарубежного программного обеспечения в деятельности предприятий Российской Федерации.

Существующие методы решения. В рамках осуществлённого научного исследования было проанализировано значительное количество методологий (любых указаний, представленных в различной форме) по проектированию архитектуры предприятия и её функционального представления, как важнейшей составной части - функциональной модели предприятия. В существующих методологиях, представленных в современной научно-методической литературе основной акцент делается или на разработке высокоуровневых обобщённых абстрактных моделей предприятий (Захман, FEAF, Gartner и т.д.) или же на наглядном отображении конкретных бизнес-процессов (в нотациях IDEF0, BPMN, еЕРС и т.д., с использованием программных средств Business Studio, ARIS, Archimate). Однако высокоуровневые обобщенные референтные модели содержат только перечень укрупненных функциональных областей (групп бизнес-процессов), а графическое моделирование бизнес-процессов (в любой нотации) целесообразно только в том случае, если перечень бизнес-процессов уже идентифицирован с достаточной степенью полноты и подробности (например, в случае, когда уже спроектирована система бизнес-процессов конкретного промышленного предприятия). Таким образом, несмотря на разнообразие существующих методологий в них не представлено конкретных указаний по разработке первоочередного компонента архитектуры предприятия - системы бизнес-процессов для конкретного крупного промышленного предприятия с необходимой степенью полноты и подробности за обозримый временной период с использованием максимально стандартных средств. То есть, в них не содержатся методы $u$ инструменты по осуществлению перехода от вы- сокоуровневых абстрактных референтных функцзиональных моделей промышленных предприятий (например, включающих функциональные области (ФО) “Снабжение”, “Производство”, “Маркетинг” и т.д.) $к$ полной, подробной и прозрачной системе простых бизнес-процессов конкретного хозяйствующего субъекта на нижнем уровне. А ведь именно система простых бизнес-процессов необходима для упорядоченного представления деятельности предприятия и повышения эффективности функционирования. Дополнительную сложность создает то, что в научно-методической литературе высокоуровневые функциональные области, как правило, называются бизнес-процессами, хотя они являются, по сути, сложными совокупностями различных работ, которые включают как более простые функциональные области, так и собственно бизнес-процессы и отдельные функции (подробнее см. в [5]).

Целью данного исследования было предложение комплекса методов и разработка формализованной методики для проектирования подробной, полной и прозрачной системы простых бизнес-процессов конкретного хозяйствующего субъекта (предприятия) на нижнем уровне. Такая система бизнес-процессов должна обязательно позволять не только создавать фундаментальную основу архитектуры предприятия для дальнейшей комплексной стандартизации и автоматизации, но и давать возможность за обозримый временной период существенно повысить экономическую эффективность функционирования современных промышленных предприятий за счёт минимизации разных видов потерь (затрат) благодаря упорядочиванию деятельности работников и совершенствованию всей системы регламентирующей документации.

Подчеркнем, что разработка системы бизнес-процессов для конкретного промышленного предприятия должна осуществляться не произвольным, а заданным образом, чтобы удовлетворять следующим ключевым требованиям:

- разработанная система бизнес-процессов должна быть основой для формализации, регламентации и автоматизации всех важных работ промышленного предприятия;

- поэтому данная система должна охватывать все работы, осуществляемые на предприятии (без дублирования и “белых пятен”);

- это требует разработки специализированной методики, позволяющей переходить от 
абстрактных референтных функциональных моделей высокого уровня к системе бизнес-процессов конкретного хозяйствующего субъекта;

- для снижения субъективности разработчиков методика должна быть представлена в максимально формализованном виде и обеспечивать построение стандартизированной системы бизнес-процессов;

- разработанная после применения методики система должна состоять из простых бизнес-процессов, которые будут нормально восприниматься всеми сотрудниками предприятия (в том числе не имеющими специальных знаний и опыта в сфере моделирования деятельности хозяйствующих субъектов), что в свою очередь, позволит упорядочить деятельность и осуществить совершенствование системы регламентирующей документации предприятия, а также дальнейшую автоматизацию;

- структура разработанной системы бизнес-процессов должна быть также понятна для рядовых работников предприятия, как и для топ-менеджеров и владельцев бизнеса.

Результаты исследования. На основе проведённого научного исследования было выявлено, что непротиворечиво и полно представить всю деятельность промышленного предприятия (особенно крупного) в виде системы бизнес-процессов за обозримый временной период можно только с использованием метода иерархической декомпозиции на основе классификации. Это обусловлено тем, что только данный метод позволяет получить прозрачную систему бизнес-процессов предприятия с необходимой степенью полноты и подробности без пересечений и пробелов и с учётом адаптации к специфическим особенностям конкретного предприятия. То есть, разработку системы бизнес-процессов промышленного предприятия необходимо осуществлять, используя концептуальный подход “сверху-вниз”, постепенно конкретизируя высокоуровневую референтную функциональную модель путём разделения функциональных областей верхнего уровня на части. При этом для повышения объективности данного деления (процесса моделирования), то есть снижения зависимости от опыта и квалификации конкретного разработчика, необходимо использовать специальные классификаторы.

Выбор базовой референтной модели. Для применения выбранного метода необходимо, в первую очередь, определиться с фундамен- тальной основой для дальнейшей разработки системы бизнес-процессов - высокоуровневой абстрактной типовой референтной функциональной моделью промышленного предприятия, иерархическая декомпозиция которой будет в дальнейшем осуществляться. В рамках настоящего научного исследования было проанализировано 10 типовых референтных функциональных моделей (например, PRM, Портера, ENAPS, IRIS, Шеера и т.д.), которые ориентированы именно на деятельность промышленных предприятий. В результате анализа и сравнительной характеристики для дальнейшей иерархической декомпозиции на базе классификации была выбрана стандартная референтная функциональная модель промышленного предприятия PRM (см. рисунок 1).

Структурирование базовой модели. Выбранная типовая референтная функциональная модель промышленного предприятия PRM иерархически декомпозируется “сверху-вниз” от общего к частному путём применения авторской формализованной методики, представленной в виде основной наглядной графической диаграммы в нотации IDEF0 (см. рисунок 2) и специализированного инструмента - классификатора работ, принцип формирования которого приведен на рисунке 3.

Иерархическая декомпозиция на основе классификации на различные виды функциональных единиц (логически идентифицируемых работ) производится последовательно от функциональных областей (ФО) идентифицированных на верхнем уровне выбранной базовой типовой референтной функциональной модели PRM по однородности и одинаковому предназначению результатов работ (например, ФО “Снабжение”), к меньшим по размеру функциональным подобластям (ФПО) разных уровней конкретизации, выделяемым из рассматриваемой ФО по определённому признаку и простым бизнес-процессам (БП), содержащим не более 25 операций. Этот процесс представлен на диаграмме, иллюстрирующей авторскую методику.

Таким образом, использование предложенной методики и инструментов позволяет получить упорядоченный и полный перечень функциональных единиц промышленного предприятия - систему бизнес-процессов, являющуюся базисом для повышения эффективности и автоматизации деятельности.

B результате практического применения 


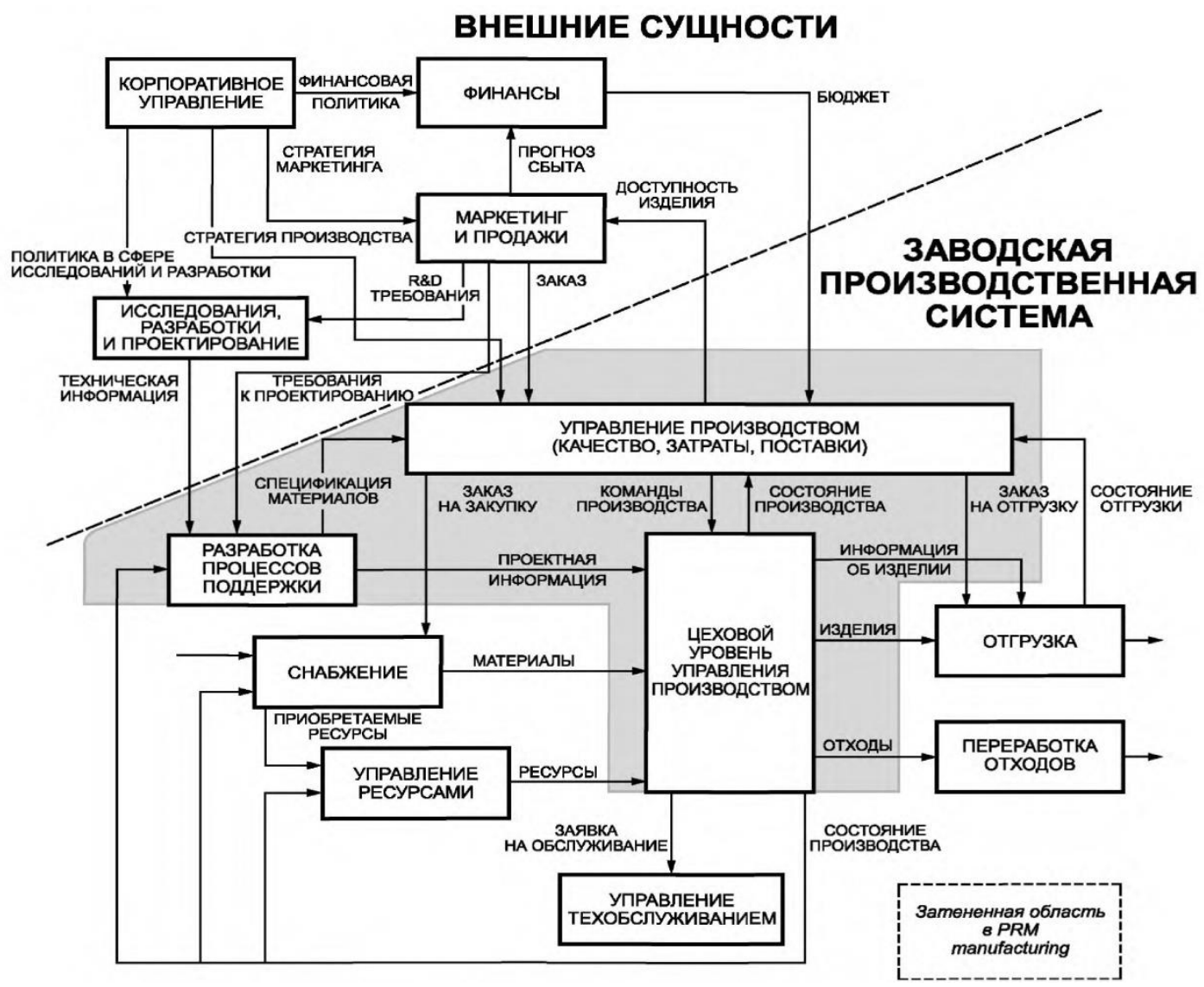

Puc. 1. Типовая референтная функциональная модель PRM (Perdue Reference Model) [6]

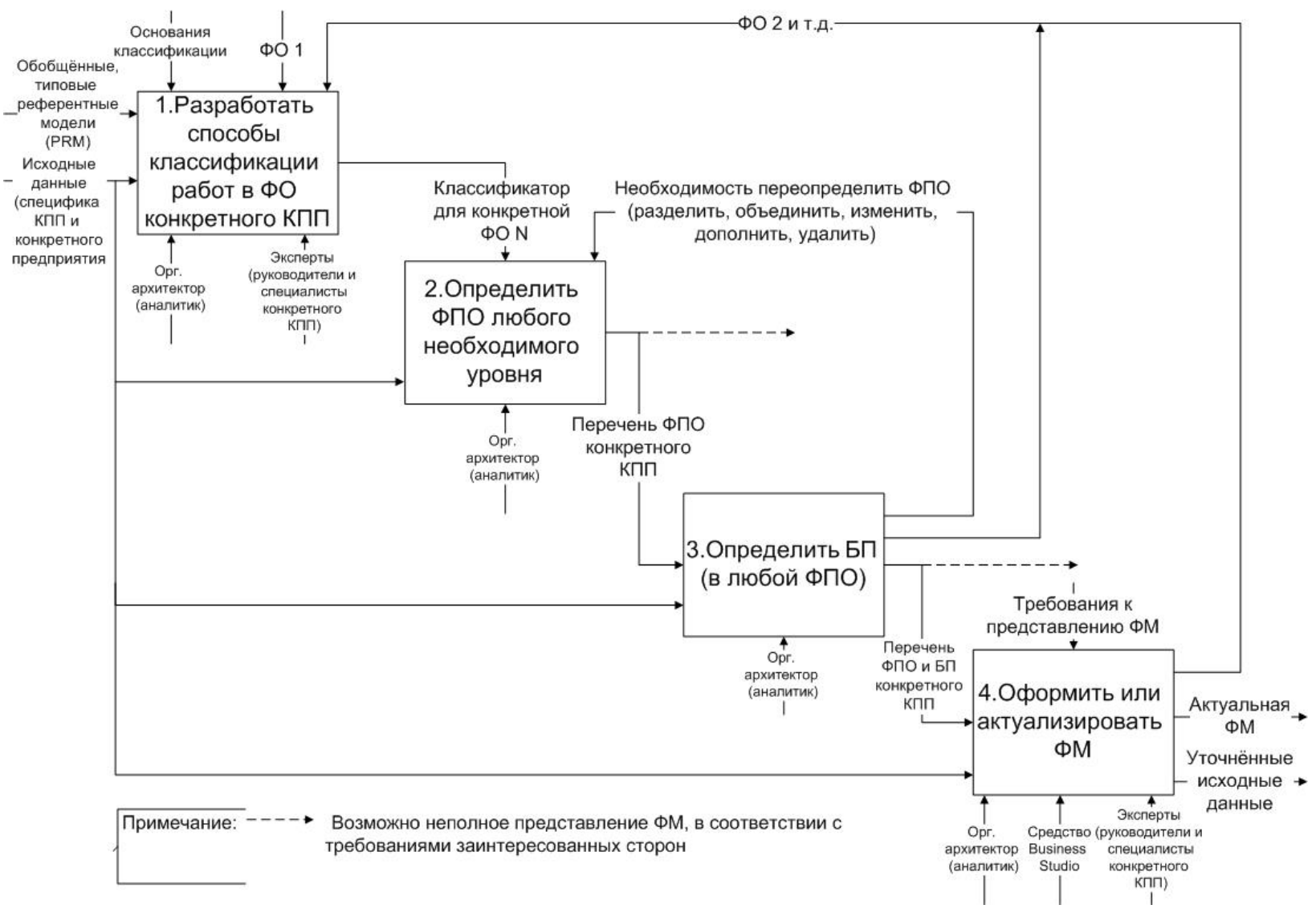

Puc. 2. Основная диаграмма процесса создания функциональной модели [7] 


\section{5.ВИД УПРАВЛЯЮЩЕГО ВОЗДЕЙСТВИЯ (Control)}

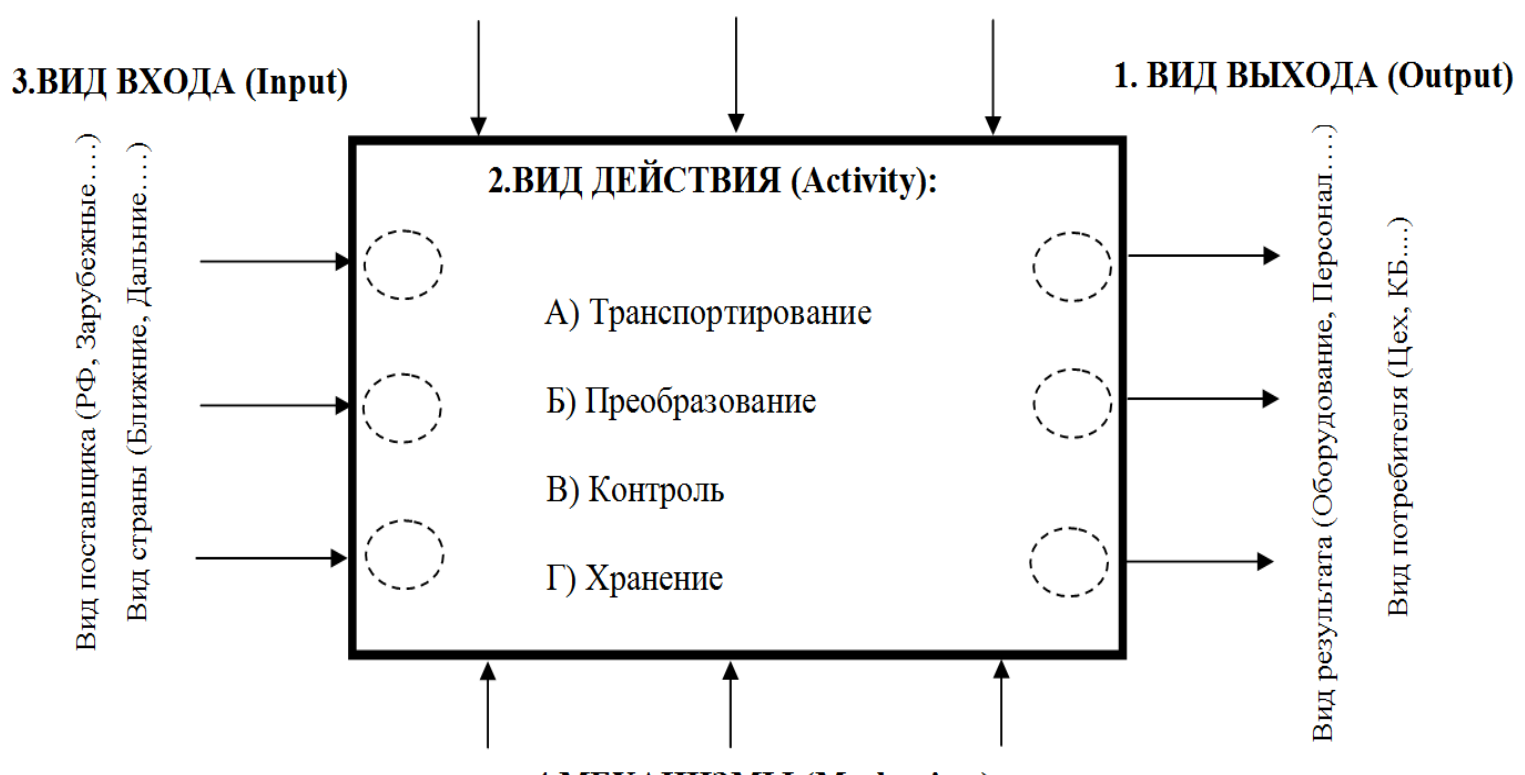

4.МЕХАНИЗМЫ (Mechanism)

Рис. 3. Принцип формирования классификатора

Источник. Разработано автором.

FА1. СНАБЖЕНИЕ

\begin{tabular}{|c|c|c|c|c|}
\hline A1 & & & бecn & печение материалами” \\
\hline & & & BP1 & $\begin{array}{l}\text { Сформировать требования к работе, при осуществлении } \\
\text { которой требуется материал }\end{array}$ \\
\hline & & & BP2 & Сформировать требования к характеристикам материала \\
\hline B1 & & & & Изделия гражданского назначения (народно-хозяйственная продукция) \\
\hline $\mathrm{C}$ & & & & Постоянно осуществляемая или периодическая закупка \\
\hline & D1 & & & Массовая поставка, крупные, средние или малые партии \\
\hline & & E1 & & Отечественные поставщики \\
\hline & & & BP1 & Приобрести и получить материал \\
\hline & & E2 & & Иностранные поставщики \\
\hline & & & BP1 & Приобрести и получить материал \\
\hline & D2 & & & Единичные поставки \\
\hline & & E1 & & Отечественные поставщики \\
\hline & & & BP1 & Найти и проверить поставщика \\
\hline & & & BP2 & Приобрести и получить материал \\
\hline & & E2 & & Иностранные поставщики \\
\hline & & & BP1 & Найти и проверить поставщика \\
\hline & & & BP2 & Приобрести и получить материал \\
\hline B2 & & & & Изделия специального назначения (оборонная, военная техника) \\
\hline $\mathrm{C}$ & & & & Постоянно осуществляемая или периодическая закупка \\
\hline & D1 & & & Массовая поставка, крупные, средние или малые партии \\
\hline & & E1 & & Отечественные поставщики \\
\hline & & & BP1 & Приобрести и получить материал \\
\hline & & & & и т. д. \\
\hline
\end{tabular}

Puc. 4. Фрагмент функциональной модели «как надо» в функциональной области “Снабжение” (авторская разработка) 
методики и классификатора разработана максимально стандартным образом система бизнес-процессов “как надо” в выбранной функциональной области “Снабжение” для конкретного известного крупного промышленного предприятия АО “ЛОМО”, г. Санкт-Петербург (фрагмент этой функциональной области приведен на рисунке 4).

Данный перечень содержит стандартизированную прозрачную систему простых коротких бизнес-процессов (включающих не более 3-5 этапов по 4-5 операций в каждом), которые потом можно доводить до графических моделей, например, в нотации IDEF0 с использованием отечественного программного средства Business Studio.

Выводы. Таким образом, предложено ре- шение актуальной научной задачи по проектированию полной и прозрачной системы бизнес-процессов для конкретного промышленного предприятия за счёт использования высокоуровневых абстрактных референтных функциональных моделей и их постепенной иерархической декомпозиции “сверху-вниз" на основе классификации. Решение данной научной задачи существенно повышает объективность процесса моделирования (снижает зависимость от лиц, которые принимают решение), а также является прочной основой для осуществления проектов по дальнейшей автоматизации и непрерывному инжинирингу деятельности промышленных предприятий для поддержания их конкурентоспособности и повышения эффективности.

\section{Библиографический список}

1. ГОСТ Р ИСО 15704-2008 «Промышленные автоматизированные системы. Требования к стандартным архитектурам и методологиям предприятия».- М.: Стандартинформ, 2010.-50 с., С. 2

2. Федеральный закон от 24.07.2007 N209-Ф3 (ред. от 29.06.2015) “О развитии малого и среднего предпринимательства в Российской Федерации” [Электронный ресурc] - URL: http://www.consultant.ru/document/ cons_doc_LAW_52144/ (Дата обращения: 01.10.2018)

3. Постановление правительства РФ от 13 июля 2015 г. № 702 “О предельных значениях выручки от реализации товаров (работ, услуг) для каждой категории субъектов малого и среднего предпринимательства” [Электронный ресурс] - URL: http://www.consultant.ru/document/ cons_doc_LAW_182963/ (Дата обращения: 01.10.2018)

4. ГОСТ Р МЭК 62264-1-2014 «Интеграция систем управления предприятием. Часть 1. Модели и терминология».- М.: Стандартинформ, 2016. - 73 с., С. 7

5. Бурмистров А.Н. О терминологии и основных положениях методологии разработки функционального представления архитектуры предприятия // Стратегическое управление организациями: технологии управления: сборник науч. тр. научной и учебно-практической конференции. Санкт Петербург. 2017. С. $14-27$.

6. ГОСТ Р МЭК 62264-1-2010 «Интеграция систем управления предприятием. Модели и терминология». Москва. 2014. С. 68

7. Штейнгарт Е.А. Бурмистров А.Н. Основные положения методики разработки архитектуры крупного промышленного предприятия и пример её применения // Экономические науки. 2017. № 1(146). С. 47-61., с. 54 